\title{
LA PRODUCCIÓN CIENTÍFICA EN LA UNIVERSIDAD ECUATORIANA: UNA PERSPECTIVA SOBRE LA DIFUSIÓN DE CONOCIMIENTO NACIONAL, REGIONAL Y MUNDIAL
}

\section{SCIENTIFIC PRODUCTION IN ECUADORIAN HIGHER EDUCATION: A PERSPECTIVE TO NATIONAL, REGIONAL AND GLOBAL KNOWLEDGE DIFFUSION}

\author{
Eco. Segundo Froilán Méndez V. MSc \\ fsmendezv@ucacue.edu.ec \\ Universidad Católica de Cuenca \\ Universidad Católica de Cuenca \\ Ing. Rafael García Abad. MSc \\ regarciaa@ucacue.edu.ec \\ Ing. Juan Carlos Ortega Castro. MSc \\ jcortegac@ucacue.edu.ec
}

\section{RESUMEN}

La difusión del conocimiento generado en los diferentes procesos de investigación constituye una preocupación latente para la Universidad Ecuatoriana. Hacer frente a la realidad regional y global respecto a producción científica, parece un horizonte aún lejano de alcanzar. Esta perspectiva vista desde las estadísticas reportadas sobre el volumen de publicación de las diferentes Instituciones de Educación Superior en el país pone en alerta sobre la importancia de encontrar estrategias necesarias que permitan la generación de conocimiento a ser diseminado. Ecuador se ubica en el puesto 95 dentro del ranking de publicaciones registrado por Scimago Journal \& Country Rank (SJR), con un total de 7942 publicaciones en el período 1996 - 2015 . El reto a vencer incluye a los diferentes actores del Sistema de Educación Superior, quienes son los responsables de propiciar las iniciativas necesarias que terminen plasmando resultados en términos de escritura científica y académica.

Palabras Clave: Producción Científica, Artículos científicos, Publicación, Revistas Indexadas

\begin{abstract}
Diffusion of knowledge generated in the different research processes is a permanent concern for Ecuadorian Higher Education. To face the regional and global reality regarding to scientific production seems to be a distant horizon to reach. This perspective, seen from reported statistics about the volume of publications from the different Universities in Ecuador, sets the alert about how important is to generate the necessary strategies that will allow knowledge to be disseminated. Ecuador is ranked 95 in the statistics shown by Scimago Journal \& Country Rank (SJR), with a total of 7942 articles that have been published between 1996 and 2015'. The challenge to beat includes the different actors of the Higher Education System, who are responsible of propitiating the required initiatives that will end showing results in terms of academic and scientific writing.
\end{abstract}

Keysword: Scientific Production, Scientific Articles, Publishing, Indexed Magazines

Recibido: julio de 2016 Aprobado: octubre de 2016

\section{Introducción}

La educación superior en el Ecuador ha sufrido cambios sustanciales en la última década, contando con un nuevo marco legal y regulatorio, situación que implica distintos requerimientos que cumplir en función de las expectativas generadas por las Universidades a nivel regional y mundial.

En la sociedad del conocimiento, la difusión del mismo representa el aseguramiento de que la dinámica de las ciencias seguirá siendo constante. Generar, transmitir y retroalimentar en las sociedades el conocimiento presente en las diferentes áreas implica el mejoramiento continuo de las capacidades de 
sus integrantes.

Como señalara Bruque (2013), "Ecuador necesita realizar un diagnóstico fiable de recursos científicos y humanos, y priorizar investigaciones"2 si se quiere dar un salto cuantitativo hacia una cultura que permita ganar visibilidad en el contexto investigativo internacional.

Las estadísticas de publicación son claras, 7942 publicaciones con Ecuador como país de origen en revistas científicas con filiación a Scimago Journal \& Country Rank, en el período comprendido entre 1996 y 2015. 1418 publicaciones en el año 2015, 993 en 2014 y 739 en 2013 en relación a publicaciones vinculadas con SJR ${ }^{3}$. 132 revistas en catálogo, 512 revistas en directorio y 82 revistas online indexadas en producción regional en cuanto a Latindex se refiere ${ }^{4}$.

Uno de los retos principales de los docentes investigadores en la Universidad Ecuatoriana viene dado por la tarea de dar a conocer los resultados de su labor. Espinosa (2010), señaló que "escribir en revistas especializadas es una de las labores académicas más relevantes en el quehacer del investigador, esta escritura puede ser con el propósito de divulgar o de difundir un conocimiento nuevo"5.

La estructura investigativa de la Educación Superior necesita priorizar la difusión y divulgación del conocimiento científico en términos de aportar al avance de la ciencia en sus diferentes esferas, más cómo hacerlo se plantea como la incógnita a ser develada. La capacitación docente debe estar ligada a la conceptualización del proceso investigativo como parte del quehacer diario del profesor universitario, caso contrario éste se convierte en una actividad esporádica que se realiza solo con el afán de llenar espacios de tiempo libre.

La interacción que debe estar presente entre la triada de Academia, Investigación y Vinculación, puede representar la alternativa a la generación de verdaderos programas y proyectos de investigación, cuyos productos acreditables son aquellos extractos plasmados dentro de un artículo científico, su proyección es ser publicado en una revista indexada de producción regional o internacional.

Las bases de datos científicas pertenecientes a las diferentes áreas del conocimiento son espacios que exigen el respectivo rigor científico al momento de difundir el fruto de una investigación realizada, por lo tanto la calidad que debe estar implícita en la elaboración de artículos que son sometidos a una revisión de pares debe ser un factor ineludible para un investigador que se precie de serlo.

El presente artículo evidencia la realidad actual de la producción, divulgación y difusión científica en el Ecuador, tomando como referentes a las Universidades de alto nivel, cuyo accionar es una constante demostración de que la labor investigativa es una prioridad en su estructura, y su razón de ser. La situación puede ser revertida, si las estrategias adecuadas se adoptan como políticas que, además de brindar relevancia y visibilidad a una Institución de Educación Superior, le permitirán abrirse campo en un mundo cada vez más competitivo y en búsqueda constante de la excelencia académica.

\section{Metodología}

Las Universidades a nivel mundial, particularmente las Ecuatorianas, se encuentran atravesando procesos de evaluación de su calidad académica, científica y tecnológica. Para conocer la realidad de las Instituciones de Educación Superior del País, en función del proceso de investigación y desarrollo que emprenden, el presente artículo se despliega mediante el uso de métodos cualicuantitativos, que permiten a través del uso de información proporcionada por diferentes fuentes conocer como actualmente se encuentran dichas Instituciones en sus parámetros de producción científica, permitiendo mediante su análisis y benchmarking, determinar el estado actual y la prospectiva hacia la que se pretende escalar.

De esta manera, primeramente se investiga sobre la tendencia en publicaciones a nivel mundial en un periodo de tiempo aceptable, que permite contar con datos válidos y confiables, luego se analiza, de la misma manera, la propensión a nivel de Latinoamérica, para concluir con el análisis a nivel nacional, con el ranking de Universidades que publican en mayor número en el País.

A través de una metodología inductiva se maneja la información obtenida de las diferentes fuentes de indagación y se determina la importancia que los actores del proceso de enseñanza - aprendizaje deben dar a la producción científica nacional dentro de las Universidades para mostrarse competitivas en su esfera de actuación.

\section{Resultados y Discusión}

"La complejidad de la educación superior en la región, desde ahora y hacia su futuro, se revela en una serie de tendencias históricas y emergentes, en su heterogeneidad, en su desigualdad, pero sobre todo en el papel que pueden 
asumir las universidades públicas y algunas muy destacadas instituciones de educación superior, para construir un nuevo escenario que coadyuve al mejoramiento sustancial de los niveles de vida para sus poblaciones, y brinde la posibilidad de un mayor bienestar, democracia e igualdad desde la ciencia, la educación y la cultura”. (Dirdiksson, 2008).

En tal virtud, es necesaria la indagación sobre las tendencias en función del número de publicaciones a nivel nacional, regional y mundial; que permitan determinar la importancia que dan las Instituciones de Educación Superior a los cambios tecnológicos, culturales y de salud, que se experimentan en la sociedad, y observar cual es el impacto y la ayuda que brindan las mismas, mediante sus proyectos de investigación y desarrollo, reflejados en los diferentes artículos que presentan de acuerdo al área del conocimiento en el que se involucra.

Para esto es necesario empezar comparando la realidad ecuatoriana frente a las publicaciones a nivel mundial y regional, para saber lo débiles que estamos en el campo de producción científica y lo conveniente e importante que resulta contribuir al desarrollo nacional desde la investigación y el desarrollo de proyectos multidisciplinares que desemboquen en productos de alto impacto con resultados que aporten al mejoramiento en los diferentes campos de acción de las Universidades. "La calidad de las revistas se define principalmente por el cumplimiento de un conjunto de pautas editoriales, de presentación, de gestión y de contenidos que garanticen el rigor científico de los artículos publicados en ellas, y por tanto abonen a su prestigio. Su visibilidad se estima, tanto por el alcance de la difusión de las revistas como por la recepción que la comunidad científica dispensa a los artículos publicados en ellas, a través de la citación". (Miguel, 2011)

En este contexto, las Universidades necesitan también mejorar sus índices de visibilidad y difusión de artículos, productos de proyectos de investigación y desarrollo, que las ubiquen en un contexto completamente competitivo, en este mundo globalizado y de mejoramiento continuo, en plena era del conocimiento.

Comparando la realidad de la producción científica ecuatoriana con el mundo, es menester la ubicación en un ambiente de superación a gran escala, según datos de Scimagojr ${ }^{6}$, el Mundo está conformado por 229 países, de los cinco continentes que han realizado algún tipo de documentación científica, entonces se puede observar la diferencia real, entre los países top y la realidad
Latinoamérica y esencialmente Ecuatoriana

Para la tabla 1 se tomó en cuenta solo los 13 primeros países del mundo, adheridos están Brasil en el puesto 23 a nivel mundial y 1 en Latinoamérica, además es necesario incluir a Ecuador, para el objeto de estudio, el mismo que se encuentra en el puesto 82 a nivel mundial y 13 en el contexto Latinoamericano, entonces se desprende una cifra de artículos indexados de 100, en el caso Ecuatoriano, con el 3\% de aportación vs 1.648 que son de los Estados Unidos, con el 15\%.

Es decir, tomando en cuenta solo un total estos 15 países que forma un universo de 100, si la muestra sería los 229 países, Ecuador tendría una aportación de alrededor del 0,5\%, expresando los valores en porcentajes. Representados en la figura 1.

\section{Ranking Mundial con más publicaciones} indexadas, 2009 - 2014

\begin{tabular}{|c|c|c|c|}
\hline \multirow{2}{*}{ Posición } & Nombre & \multicolumn{2}{c}{$\begin{array}{c}\text { \# Documentos } \\
\text { Científicos }\end{array}$} \\
\hline 1 & $\begin{array}{c}\text { Estados } \\
\text { Unidos }\end{array}$ & 3.485 .382 & 1.648 \\
\hline 2 & Reino Unido & 1.008 .632 & 1.015 \\
\hline 3 & Alemania & 903.368 & 887 \\
\hline 4 & Francia & 646.740 & 811 \\
\hline 5 & Canadá & 538.796 & 794 \\
\hline 6 & Japón & 747.124 & 745 \\
\hline 7 & Italia & 535.146 & 713 \\
\hline 8 & Holanda & 303.543 & 694 \\
\hline 9 & Suiza & 222.286 & 686 \\
\hline 10 & Australia & 438.518 & 644 \\
\hline 11 & Suecia & 192.261 & 614 \\
\hline 12 & España & 460.668 & 591 \\
\hline 13 & Bélgica & 167.681 & 547 \\
\hline 23 & Brasil & 329.922 & 379 \\
\hline 82 & Ecuador & 3.662 & 100 \\
\hline
\end{tabular}

Tabla 1.

Fuente: Producción Científica del Mundo, www. scimagojr.com. 


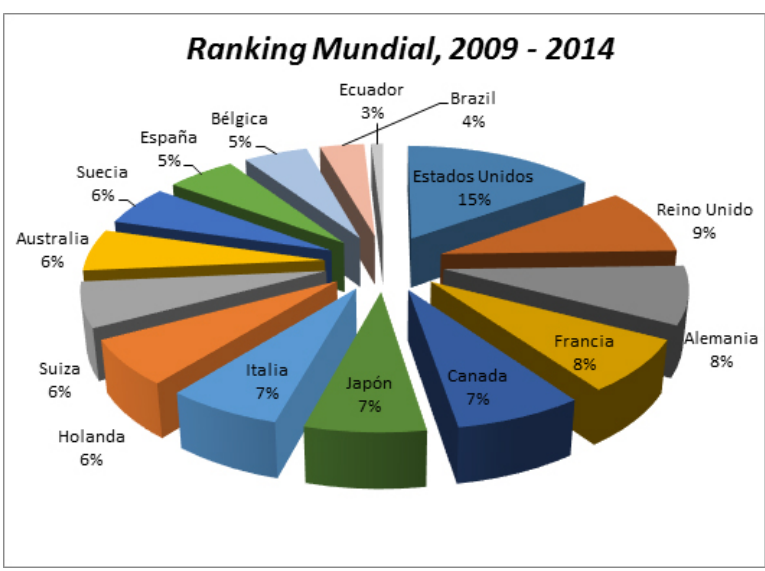

Figura 1. Tabulación publicación científica a nivel mundial Fuente: El presente artículo

Si bien es necesaria la contextualización del ámbito nacional, no resulta menos necesaria la comparación que tiene la Universidad Ecuatoriana en relación a la Región, sabiendo que Latinoamérica y el Caribe cuenta con 48 países sumados los países dependientes con el idioma castellano y otras lenguas, según datos de Scimagojr, entonces la productividad científica de la Universidades de la Región va depender de varios factores endógenos, como: la cultura científica que tiene cada país, la estructura educativa superior, etc. Misma que se puede observar en la tabla 2.

Por otro lado se encuentran, los factores exógenos que están intrínsecos en un mundo globalizado con corrientes tecnológicas avanzadas y porque no decir, la influencia de valores, oportunidades de países vecinos con mayor y mejor estructuración sobre el conocimiento científico.

Para este análisis, se tomó en cuenta solo los 15 primeros países de Latinoamérica, de donde se obtiene que el Ecuador produce un $4 \%$ para un universo de los 15 que es el $100 \%$, tomando en cuenta los 48 países, el Ecuador aportaría alrededor del $2 \%$. Reflejados en la figura 2.

Ranking de Países de Latinoamérica con más publicaciones indexadas, 2009 - 2014

\begin{tabular}{|c|c|c|c|}
\hline Posición & Nombre & $\begin{array}{l}\text { \# Documentos } \\
\text { Científicos }\end{array}$ & \# H. Index \\
\hline 1 & Brasil & 329.922 & 379 \\
\hline 2 & México & 101.946 & 289 \\
\hline 3 & Argentina & 67.091 & 273 \\
\hline 4 & Chile & 48.776 & 233 \\
\hline 5 & Colombia & 34.666 & 169 \\
\hline 6 & Puerto Rico & 5.196 & 156 \\
\hline 7 & Venezuela & 12.013 & 155 \\
\hline 8 & Perú & 7.413 & 140 \\
\hline 9 & Panamá & 2.469 & 128 \\
\hline
\end{tabular}

\begin{tabular}{|c|c|c|c|}
\hline 10 & Costa Rica & 3.848 & 127 \\
\hline 11 & Uruguay & 6.354 & 124 \\
\hline 12 & Cuba & 12.594 & 115 \\
\hline 13 & Ecuador & 3.662 & 100 \\
\hline 14 & Bolivia & 1.528 & 78 \\
\hline 15 & $\begin{array}{c}\text { Trinidad y } \\
\text { Tobago }\end{array}$ & 2.254 & 72 \\
\hline
\end{tabular}

Tabla 2.

Fuente: Producción Científica de Latinoamérica, www.scimagojr.com.

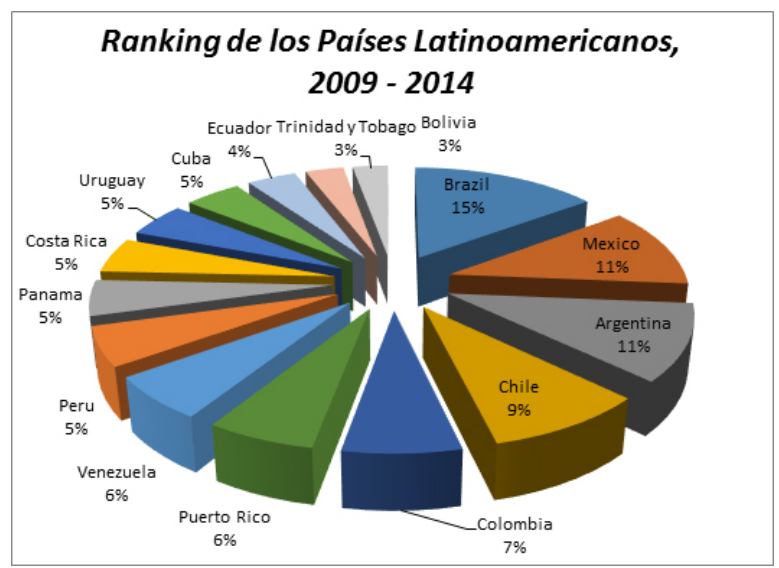

Figura 2. Tabulación publicación científica a nivel de Latinoamérica

Fuente: El presente artículo

En el contexto nacional, tabla 3, se puede aportar que, el Ecuador cuenta con 51 Universidades categorizadas como A,B,C y D, según datos de la Senescyt ${ }^{7}$, entonces la productividad científica de éstas universidades va depender de varios factores, mismos que son la diferencia entre las ubicadas en los primeros lugares, como la Universidad San Francisco de Quito, que hacen uso de un gran apoyo económico, infraestructura, visibilidad de revistas indexadas, docente con el tiempo estandarizado para la elaboración de artículos científicos entre otros, todo esto en relación con las Universidades con escasa documentación científica.

Para esto, se ha recopilado información desde el año 2009 hasta el 2013 con el objetivo de conocer y establecer una proyección ascendente de la producción científica de la Universidad Ecuatoriana en un futuro cercano, en tal virtud, de este cuadro se calcularon solamente las 15 primeras Universidades del Ecuador para un universo de 100, expresadas en porcentajes, figura 3. 
Ranking de las Universidades del Ecuador con más publicaciones indexadas, 2009 - 2013

\begin{tabular}{|c|c|c|c|}
\hline Posición & Nombre & $\begin{array}{l}\text { \# Publicacio- } \\
\text { nes Científicas } \\
\text { SCOPUS }\end{array}$ & $\begin{array}{c}\% \text { Publica- } \\
\text { ciones Alta } \\
\text { Calidad }\end{array}$ \\
\hline 1 & $\begin{array}{c}\text { Universidad San } \\
\text { Francisco de } \\
\text { Quito USFQ }\end{array}$ & 422 & 74,17 \\
\hline 2 & $\begin{array}{c}\text { Pontificia } \\
\text { Universidad } \\
\text { Católica del } \\
\text { Ecuador }\end{array}$ & 319 & 53,61 \\
\hline 3 & $\begin{array}{c}\text { Escuela Politéc- } \\
\text { nica Nacional } \\
\text { EPN }\end{array}$ & 152 & 61,18 \\
\hline 4 & $\begin{array}{c}\text { Universidad } \\
\text { Técnica Parti- } \\
\text { cular de Loja } \\
\text { UTPL }\end{array}$ & 143 & 31,47 \\
\hline 5 & $\begin{array}{c}\text { Escuela Supe- } \\
\text { rior Politécnica } \\
\text { del Litoral } \\
\text { ESPOL }\end{array}$ & 137 & 32,85 \\
\hline 6 & $\begin{array}{l}\text { Universidad } \\
\text { Central del } \\
\text { Ecuador UCE }\end{array}$ & 103 & 40,78 \\
\hline 7 & $\begin{array}{c}\text { Universidad } \\
\text { de Cuenca U } \\
\text { Cuenca }\end{array}$ & 91 & 42,86 \\
\hline 8 & $\begin{array}{c}\text { Universidad } \\
\text { Católica de } \\
\text { Santiago de } \\
\text { Guayaquil }\end{array}$ & 90 & 33,33 \\
\hline 9 & $\begin{array}{c}\text { Universidad } \\
\text { de las Fuerzas } \\
\text { Armadas ESPE }\end{array}$ & 53 & 37,74 \\
\hline 10 & $\begin{array}{l}\text { Universidad } \\
\text { Tecnológica In- } \\
\text { doamérica UTI }\end{array}$ & 42 & 42,86 \\
\hline 11 & $\begin{array}{l}\text { Universidad de } \\
\text { Especialidades } \\
\text { Espíritu Santo }\end{array}$ & 34 & 26,47 \\
\hline 11 & $\begin{array}{l}\text { Universidad de } \\
\text { Guayaquil UG }\end{array}$ & 34 & 44,12 \\
\hline 12 & $\begin{array}{l}\text { Universidad } \\
\text { Politécnica Sa- } \\
\text { lesiana UPS }\end{array}$ & 32 & 6,25 \\
\hline 13 & $\begin{array}{c}\text { Universidad } \\
\text { Nacional de } \\
\text { Loja UNL }\end{array}$ & 29 & 48,28 \\
\hline 14 & $\begin{array}{l}\text { Universidad de } \\
\text { Las Américas } \\
\text { UDLA }\end{array}$ & 25 & 32,00 \\
\hline 15 & $\begin{array}{l}\text { Universidad } \\
\text { Andina Simón } \\
\text { Bolívar UASB }\end{array}$ & 22 & 9,09 \\
\hline 14 & Bolivia & 1.528 & 78 \\
\hline
\end{tabular}

Tabla 3

Fuente: Producción Científica del Ecuador Secretaria Nacional de Educación Superior, Ciencia y Tecnología.

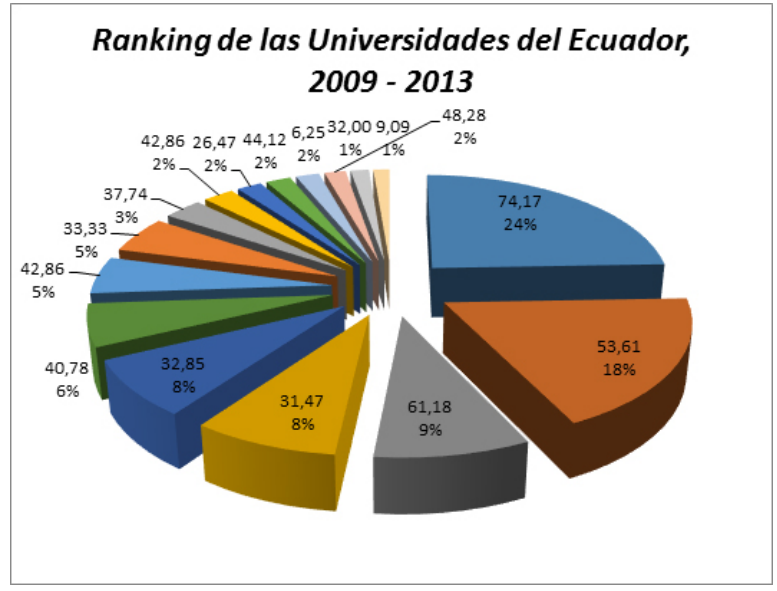

Figura 3. Tabulación publicación científica del Ecuador

Fuente: El presente artículo

Logrando de esta manera mostrar las tendencias en publicaciones a nivel mundial, y sobre todo el sitio en el cual se encuentran las Universidades ecuatorianas, frente a las de la región y el mundo, y cuáles de ellas tienen mayor porcentaje de publicaciones dentro de su ámbito de desarrollo, permitiendo reflejar que aún nos queda mucho por hacer y que la principal ventaja que se puede obtener de esta información es que la vara es muy alta, pero completamente alcanzable, mientras mayor sea el interés de mostrarnos hacia el mundo con nuestra producción científica y el desarrollo de proyectos de investigación que coadyuven al avance tecnológico y social, dentro del campo de la Educación Superior.

\section{Conclusiones}

Mediante el benchmarking relacionado con la calidad de producción científica de las Universidades a nivel nacional, regional y mundial, se concluye:

- La información recopilada muestra la prospectiva respecto a la realidad actual de las Universidades ecuatorianas con contrastada con las de Latinoamérica, mostrando la debilidad que tienen éstas en relación a su producción científica y la importancia que tiene la misma, evidenciando la necesidad de mejorar sus índices de calidad y competitividad dentro del ámbito de la construcción y divulgación del conocimiento.

- Es fundamental contar con datos precisos que permitan tomar decisiones en función del mejoramiento continuo de la cultura investigativa, y el cambio de mentalidad que se pretende fomentar en los docentes universitarios, sobre la relevancia de publicar sus experiencias, en los diferentes ámbitos y áreas en las que se desempeñan, dentro de su quehacer académico, no solamente en proyectos de investigación y desarrollo, sino también en función de la información manejada 
en cada una de sus funciones.

- Estas tendencias a nivel nacional, regional y mundial, y su impacto, reflejan de forma significativa lo que al pasar de los años las universidades han tomado como eje de funcionamiento, y han dado prioridad a sus procesos de investigación, y la difusión de sus resultados, conocedores de que mientras más visibles sean para el mundo, mejores resultados obtendrán es sus parámetros de calidad, aceptación y respeto académico.

\section{Referencias bibliográficas}

1. Albornoz, M. Política Científica y Tecnológica. Una visión desde América Latina. Buenos Aires: CICTES; 2001.

2. Bonder, G. Equidad de género en ciencia y tecnología en América Latina: bases y proyecciones en la construcción de conocimientos, agendas e institucionalidades. Washington: UNESCO; (2004)

3. Didriksson, A, Contexto global y regional de la Educación Superior en América Latina y El Caribe, México; 2008.

4. Fernández Lamarra, N. Hacia una nueva agenda para la educación superior: internacionalización, evaluación de la calidad y educación virtual, Virtual Educa 2004, Fórum de Barcelona, p. 11; (2004).

5. Gutiérrez, C; Mayta, P. Publicación desde el Pre Grado en Latinoamérica: Importancia, Limitaciones y Alternativas de Solución. Lima: CIMEL; 2003.

6. Miguel, S, Revistas y producción científica de América Latina y el Caribe: su visibilidad en SciELO, RedALyC y SCOPUS, Argentina: Revista Interamericana de Bibliotecología; 2011

7. SCImago Jornal \& Country Rank. (2016). http:// www.scimagojr.com/.

8. Secretaría de Educación Superior, Ciencia Tecnología e Innovación. (2016). http://www.educacionsuperior.gob.ec/.

9. Walsh, C. La interculturalidad y la educación básica ecuatoriana: propuestas para la reforma educativa. Quito: Revista Ecuatoriana de Historia; 1998.

\section{Citas}

1 Scimago Journal \& Country Rank (2016). http:// www.scimagojr.com/countryrank.php

2 Bruque, S (2013): La producción científica en Ecuador en el contexto latinoamericano, http:// www.senescyt.gob.ec/adjuntos/SEMINARIO HERRAMIENTAS_CIENTIFICAS/5\%20Sebastian $\% 20$ Bruque $\% 20$ Producion $\% 20$ cientifica $\% 20$ en\%20Ecuador.pdf. Fecha de consulta: 26 de abril de 2016.
3 Scimago Journal \& Country Rank (2016). http:// www.scimagojr.com/countryrank.php?year=2015

4 Índices estadísticos de producción regional, Latindex (2016). http://www.latindex.org/latindex/ tablaPais? $\mathrm{id}=16$ \&id2=2

5 Espinosa, V (2010): Difusión y Divulgación de la Investigación Científica, http://www. scielo.cl/scielo.php?script=sci_arttext\&pi$\mathrm{d}=$ S0718-34292010000300001. Fecha de consulta: 26 de abril de 2016

6 SCImago Journal \& Country Rank. http://www. scimagojr.com/

7 Secretaría de Educación Superior, Ciencia Tecnología e Innovación. http://www.educacionsuperior.gob.ec/ 